Ebisu Ebisu

Études japonaises Études japonaises

55 | 2018

La fabrique des villes japonaises contemporaines

\title{
Le regard urbain fondu dans les médias
}

メディアに浴解する都市のまなざし

Media and the Urban Gaze

\section{Shun.ya Yoshimi}

Traducteur : Mathieu Capel

\section{OpenEdition}

\section{Journals}

Édition électronique

URL : http://journals.openedition.org/ebisu/2797

DOI : 10.4000/ebisu.2797

ISSN : 2189-1893

Éditeur :

Institut français de recherche sur le Japon (UMIFRE 19 MAEE-CNRS), Maison franco-japonaise

\section{Édition imprimée}

Date de publication : 15 décembre 2018

Pagination : 171-184

ISSN : 1340-3656

\section{Référence électronique}

Shun.ya Yoshimi, «Le regard urbain fondu dans les médias », Ebisu [En ligne], 55 | 2018, mis en ligne le 15 décembre 2018, consulté le 01 mai 2019. URL : http://journals.openedition.org/ebisu/2797 ; DOI :

10.4000/ebisu.2797 


\section{Le regard urbain fondu dans les médias}

\section{Yoshimi Shun.ya}

メディアに溶解する都市のまなざし

吉見俊哉

Media and the Urban Gaze

Yoshimi Shun.ya

Cots-clés : Urbanisation, modernité, architecture, visualité, écrans, sociologie des médias.

Lauteure : Né en 1957, Yoshimi Shunya est professeur de sociologie à l'université de Tokyo et doyen de l'Initiative interfaculté des sciences de l'information. Spécialiste d'études urbaines, culturelles et médiatiques, il est l'auteur de nombreux ouvrages parmi lesquels Dramaturgie de la ville - Tokyo, histoire sociale de ses quartiers animés (1987), Politique de l'exposition - la modernité du regard (1992), Capitalisme de la "voix" (1995).

Résumé : Extrait de Géopolitique de la ville visuelle - la modernité comme regard, que le sociologue Yoshimi Shunya a fait paraitre en 2015. Sous le patronage notamment de Michel Foucault et de Jonathan Crary, Yoshimi y décrit, non pas les éléments qui auraient présidé à la reconfiguration moderne du regard, mais la manière dont la modernité serait advenue dans les villes comme autant d'expériences et de structures collectives fondamentalement innervées par les questions de distribution, d'organisation et de disciplinarisation des regards. À ce titre, l'objectif est de rendre compte d'un véritable retournement épistémologique : ou comment la ville aurait cessé de répondre de logiques architecturales, pour se conformer désormais à des exigences iconiques et médiatiques. 
キーワード

都市化、近代、建築、視覚性、スクリーン、 メディア社会学

\section{著者}

1957 年東京都生まれ。日本の社会学者。東 京大学大学院情報学環教授。専門は文化研究、 メディア研究、都市論、カルチュラル・スタ ディーズ。単著作は『都市のドラマトゥルギー

東京・盛り場の社会史』(1987)、『展覧会 の政治学 眼差しの近代』(1992)、『「声」の 資本主義——電話・ラジオ・蓄音機の社会史』 他多数。

\section{要旨}

吉見俊哉著『視覚都市の地政学』の抜粋。ミ シェル・フーコーやジョナサン・クラリーの 思想を受け継いで、吉見俊哉は視線の構造の 近代的変容ではなく、むしろ都市における近 代の経験はどのようにまなざしという経験 (つまりまなざしの再構成、再配布、規律化) に貫かれてきたか、という問いかけに基づき 都市の再編成を考察する。この意味において 本論考が目指すのは認識論的な転回である。 つまり、いかにして都市が建築のロジックに 従うことをやめて図像的な要請やメディアの 要求に順応するかを明らかにするのだ。
\ Keywords: Urbanisation, modernity, architecture, visuality, screens, media sociology.

The Author: Sociologist Yoshimi Shun.ya heads the Interfaculty Initiative in Information Studies at the University of Tokyo, where he specialises in urban, cultural and media studies. Notable works include Dramaturgy in the City: A Social History of Popular Entertainments in Modern Tokyo (1987), The Politics of Exposition (1992) and Voice of Capitalism (1995).
Abstract: This paper is an extract from Geopolitics of the Visual City (2015), published in Japanese by the sociologist Yoshimi Shunya, in which the author draws on the work of Michel Foucault and Jonathan Crary to explore not how people's gaze has been reconstructed in modern times, but how the reorganisation, redistribution and disciplining of the gaze fundamentally informs the way modernity is experienced in cities. The aim is to document a veritable epistemological change: namely, that the city has ceased being driven by architectural principles and now conforms to the demands of visual media. 


\title{
Le regard urbain fondu dans les médias
}

\author{
YOSHIMI Shun.ya*
}

\section{La ville écran}

Dès le XIX ${ }^{\mathrm{e}}$ siècle, les mouvements conjugués de l'attention et de la distraction, de la discipline (c'est-à-dire de l'instruction) et du spectacle ont émergé dans des espaces publics d'un type particulier - lieux de visualité commune où s'assemblait une foule immense et hétérogène - pour imprégner bientôt toute la ville, logés à l'intérieur même des médias ${ }^{1}$. Parmi ces lieux de visualité, les premiers à apparaître furent les différents dispositifs propres à la ville moderne, tels qu'expositions, musées d'art et d'histoire, zoos ou grands magasins. J'ai décrit dans Tenrankai no seijigaku - manazashi no kindai 展覧 会の政治学一眼差しの近代 (Politique de l'exposition - modernité du regard, 1992) les politiques d'intégration, d'opposition ou de compétition qui se sont tramées dans ce genre d'espaces publics, entre regard, pouvoir et corps collectif; mais il reste désormais à vérifier jusqu'où les politiques visuelles développées au XIX ${ }^{\mathrm{e}}$ siècle dans les espaces de l'exposition, du musée, et même des grands magasins, se sont étendues, au $\mathrm{Xx}^{\mathrm{e}}$ siècle, aux rapports

1. Cet article est la traduction d'un extrait de l'introduction de Géopolitique de la ville visuelle - la modernité comme regard『視覚都市の政治学 まなざしとしての近代』, Tokyo, Iwanami shoten 岩波書店, 2016 : 34-44. (NdR)

* Professeur à l'université de Tokyo. 
entre écran de cinéma et spectateurs, ou encore au voisinage d'un grand nombre de médiums : théâtres d'ombres, téléviseurs de rue (gaitōterebi 街頭 テレビ), vitrines ou grandes affiches des gares.

Ceux-ci étaient autant de signes que les politiques visuelles déployées jusque-là dans les espaces publics à un niveau architectural ou tridimensionnel seraient, à terme, prises en charge par des surfaces planes, bidimensionnelles, grâce à la fois aux écrans de cinéma ou de télévision, et aux dispositifs d'affichage (disupurē sōchi ディスプレー装置) allant d'immenses surfaces murales aux terminaux portables. La reproduction bidimensionnelle de la réalité qui, dans la première moitié du $\mathrm{Xx}^{\mathrm{e}}$ siècle, s'était répandue dans le monde par le biais des écrans de cinéma, a bientôt gagné les foyers avec la généralisation des téléviseurs (s’y banalisant). Plus encore depuis les années 1980 et le décloisonnement des catégories de médias, le nombre des écrans digitaux a augmenté de façon spectaculaire, des murs d'immeubles aux portables. Ōkubo Ryō 大久保遼 affirme ainsi qu'avec, [primo,] l'«omniprésence des écrans sous leurs formes diverses, outre la photographie, le cinéma et la télévision [; secundo,] l'allongement du temps passé dans notre vie en compagnie des images" [; tertio,] l'avènement d'une situation où, au-delà des seules formes dites cinématographiques et télévisuelles, «il n’est désormais plus possible de se passer, ni dans les salles de concert, ni dans les musées, ni dans les parcs à thèmes, ni même dans les lieux d'éducation et d'information, d'affaires ou de santé, des techniques de visualisation de l'information par l'image», on ne saurait plus désormais "appréhender les rapports "images/espace/corps" à partir des seuls modèles "film/salles de cinéma/spectateurs" et "télévision/foyer/téléspectateurs" " (Ōkubo 2015 : 14-15). Si, comme le montre encore Ōkubo, la visualité propre au cinéma et à la télévision, toujours dominante aujourd'hui, remonte au moins aux attractions diverses et variées qu'on trouve dans la société du début du XIX ${ }^{e}$ siècle, l'important toutefois n'est pas de raconter ce passé tel une "histoire des origines" à nos jours, mais de l'interroger du point de vue des innombrables "possibles historiques» que renferme la ville visuelle (shikaku toshi 視覚都市).

Le devenir-visuel de la ville (shikaku toshika 視覚都市化) comprend l'entrelacement de ces visions tri- et bidimensionnelles. D'un côté, s'est déroulé de la fin du $\mathrm{XIX}^{\mathrm{e}}$ siècle au milieu du $\mathrm{Xx}^{\mathrm{e}}$ un processus au cours duquel les écrans à deux dimensions ont été introduits et ont proliféré dans l'espace à trois dimensions de la ville. La similitude entre notre expérience visuelle au 
sein d'espaces urbains tridimensionnels tels qu'expositions, musées et grands magasins, et celle qui est la nôtre devant l'écran d'une salle de cinéma, serait suggérée par le «regard en mouvement» qui les sous-tend l'une et l'autre. En effet si, selon la thèse de Wolfgang Schivelbusch, les expositions proposaient bien les versions miniatures du tourisme, et les grands magasins ses formes banalisées, l'expérience qu'offraient les salles de cinéma participait en vérité d'un même «regard du voyageur ferroviaire²». Par exemple, dans les expositions universelles de Paris, de Chicago ou de Saint-Louis, les visiteurs se déplaçaient parmi des objets qui, disposés les uns à côté des autres, permettaient d'embrasser du regard l'[histoire allant de] la «civilisation" aux "temps primitifs»; mais dans une salle de cinéma, les spectateurs voyagent de même à travers les paysages de régions et d'époques différentes. Anne Friedberg le résume ainsi :

Inaugurée à la fin du XIx siècle, la projection d'images mouvantes [...] reposait sur l'immobilité du spectateur et l'ouverture d'un cadre fixe. L'association nouvellement ouvragée de visualités mobiles et virtuelles offrait une mobilité virtuelle à des spectateurs immobiles, alors témoins de mouvements confinés. En tant que "spectateurs" de cinéma, nous sommes assis sans bouger devant des images mouvantes; notre corps ne bouge pas, mais notre "point de vue" le peut en revanche. (Friedberg 2012:8)

Commentant cette mobilité du regard, Friedberg a souligné l'isomorphie de l'expérience cinématographique et du lèche-vitrine. Pour elle, ce dernier

implique [de lancer] un regard spéculatif à la mise en scène de la vitrine, sans intention d'entrer dans le magasin ni de faire un achat. Regarder un film repose de manière identique sur une contemplation distanciée : un tableau, encadré ou inaccessible, non pas derrière une vitre, mais sur un écran,

de sorte que ce regard virtuel se retrouve également chez le spectateur de cinéma (Friedberg 2008 : 84). Selon ses thèses, le regard du spectateur de cinéma prolonge non seulement celui du flâneur des passages décrit par

2. Ici, Yoshimi fait référence à Schivelbusch Wolfgang, The Railway Journey: The Industrialization of Time and Space in the Nineteenth Century, Urizen Books, 1979. (NdT) 
[Walter] Benjamin, mais se retrouve aussi dans l'œil du consommateur allant et venant dans les grands magasins et devant les vitrines. À l'évidence, on reconnait ici cette simplification abusive qu'a sévèrement critiquée Nakamura Hideyuki 中村秀之, qui "généralise à l'investissement subjectif (shutaika 主体化) du "spectateur" répondant à l'appel du texte filmique celui, "similaire", du "consommateur" (Nakamura 2010 :122) - c'est-à-dire l'oubli de la puissance latente des images. Toutefois, pareille simplification a aussi l'avantage d'éclairer la manière dont se compose, au-delà des différentes catégories de l'espace, le champ de la «modernité comme regard".

La thèse de Friedberg a valeur heuristique surtout lorsqu'elle aborde les rapports entre images et architecture urbaine. Elle mentionne ainsi la parenté des écrans de cinéma avec les murs de verre du Crystal Palace de l'exposition universelle de Londres [en 1851]. Les salles obscures ont donné naissance à "un nouveau type de bâtiments [nécessaire] pour "héberger" l'écran de projection». Sans doute est-il injuste que nombre d'historiens de l'architecture se soient focalisés sur le lumineux bâtiment de verre et de métal de l'exposition universelle de Londres, au détriment des salles obscures des cinémas et d'autres [formes d'] architectures de la lumière apparues un demi-siècle plus tard dans les métropoles d'Europe et des États-Unis. À la faveur de "la mutation architectonique inaperçue des murs-écrans ${ }^{3}$ ", ont été érigés de nouveaux murs baignés de lumière électrique (Friedberg 2012 : 205-206), avant que ces bâtiments en forme d'écrans de projection ne recouvrent entièrement les villes $\mathrm{du} \mathrm{xx}^{\mathrm{e}}$ siècle, parallèlement aux bâtiments du type Crystal Palace. Si ceux-ci ont donc pour prolongement d'innombrables gratte-ciel, ceux-là en revanche ont proliféré, des salles de cinéma aux immenses systèmes d'affichages (disupurē ディスプレー) des quartiers les plus animés. En général, l'architecture

3. Non référencée comme telle dans le texte de Yoshimi, cette expression est en fait empruntée par Friedberg à Paul Virilio, Lost dimension, MIT Press, coll. Semiotext(e) Foreign Agents, Cambridge, 1991, 192 p., traduction en langue anglaise de La crise des dimensions : la représentation de l'espace et la notion de dimension, École spéciale d'architecture, Paris, 1983, 119 p. De même, "architectures de la lumière» est la traduction directe de l'expression "architecture of light of the dark room» employée par Friedberg. (NdT) 
implique des mouvements vers l'intérieur, à travers, vers le haut, vers le bas, vers l'extérieur. Mais en tant que spectateurs de cinéma, téléspectateurs ou utilisateurs d'ordinateurs, nous restons immobiles face à des écrans remplis d'images et de sons. Devant un écran, le spectateur/téléspectateur/utilisateur se trouve pris dans un nœud [...] de mobilité et d'immobilité [...], de matérialité et d'immatérialité [...]. [En même temps] [1]es écrans fonctionnent comme des éléments architectoniques, ouvrant dans la matérialité des espaces construits des trouées virtuelles, en une véritable "architecture de la spectatorialité». (Friedberg 2012 :102-103)

Aussi en vient-on à considérer l'histoire au cours de laquelle la ville s'est lentement fondue dans les images, au lieu d'une autre où les images se seraient lentement fondues dans la ville. Approximativement, ce processus s'est déroulé selon trois phases structurelles. Dans la première, les écrans de cinéma pénètrent la ville. Au début du $\mathrm{Xx}^{\mathrm{e}}$ siècle, différents types de salles de cinéma sont construits dans les quartiers animés, des nickelodéons peu onéreux aux grands palaces luxueux, pour gagner bientôt les rues commerçantes du voisinage. En dehors de ces salles, des écrans sont installés en certaines occasions pour projeter des films dans les cours d'école et sur les places, dans les stades ou dans l'enceinte des temples. Ici, l'écran reste "une lumière dans l'obscurité», rien de plus qu’un «lieu» donné du «territoire», un «point» sur sa " carte $^{4} »$.

La deuxième phase voit se répandre des architectures solidaires d'écrans de projection. À la fin du $\mathrm{xx}^{\mathrm{e}}$ siècle, les immeubles qui changent ainsi leurs parois en écrans gigantesques proliferent devant les gares et dans les quartiers populaires, mais pour peu qu'on leur ajoute les images mouvantes des panneaux publicitaires, le nombre des écrans extérieurs dans les grandes villes devient colossal. N'ont cessé d'augmenter également les bâtiments dont l'intérieur se couvrait d'images à mesure que s'agrandissaient les moniteurs. Depuis les années 2000 enfin, avec la propagation foudroyante des terminaux portables comme l'iPhone et l'iPad, tout le monde parcourt désormais les villes contemporaines un petit écran à la main. Ici, les écrans - parois urbaines autant que miroirs de poche - en viennent à recouvrir entièrement les surfaces de la ville envisagée comme espace architectural.

4. La phrase originale joue du terme japonais correspondant à "carte géographique», chizu 地図, qu'elle décompose en ses deux kanji : les écrans étant alors autant de $z u$ 図 (figure, dessin) localisés dans la ville envisagée comme chi 地 (terre, territoire). (NdT) 
On remarque d'ailleurs non sans intérêt que la mode d'une sémiotique de la ville, y voyant un «texte à lire», s'est très exactement superposée au début de cette deuxième phase. Des années 1970 aux années 1980, il s'est produit à l'évidence une modification structurelle dans la manière dont la visualité se tramait entre les couches superficielles des villes et le corps de leurs habitants.

Enfin, au cours d'une troisième phase, alors que ce type d'images a fini par saturer la ville, les bâtiments ont commencé d'y être conçus selon des logiques moins architecturales qu'iconiques. Inauguré en 1983, Disneyland Tokyo a été véritablement l'archétype du devenir-espace architectural des images (eizō no kenchiku kükanka 映像の建築空間化). [...] Il y a un siècle, celles-ci se sont donc répandues dans la ville par le biais des salles de cinéma, pour intégrer bientôt les murs des immeubles et les publicités en plein air, jusqu'à ce qu'enfin les bâtiments eux-mêmes agissent comme autant d'entités iconiques - chaque individu commençant en parallèle à porter sur soi des images. Il s'est agi là moins d'un processus successif, étape par étape, que de trois phases de transformation structurellement liées les unes aux autres.

\section{La ville-publicité comme point-limite et l'homogénéisation de l'espace}

S'il s'est produit à l'échelle planétaire, dans des villes telles que NewYork ou Los Angeles, jusqu’à Singapour, Mexico City ou Dubaï, dans le cas du Japon ce processus s'est développé en premier lieu à Tokyo. C'est sur elle par conséquent qu'une analyse géopolitique des images/villes doit nécessairement se concentrer. Dans Toshi no doramaturugi 都市のドラマ トゥルギー (La dramaturgie de la ville), que j'ai publié il y a environ trente ans, en 1987, j'essayais d'expliciter la "modernité comme regard», telle qu'elle est advenue dans la Tokyo moderne, en réexaminant la manière dont les regards se croisaient et les comportements se modifiaient dans les quartiers populaires d'Asakusa, Ginza, Shinjuku ou Shibuya. À l'époque, et selon des schémas approximatifs, si l'"Asakusa» des années 1910 et le "Shinjuku» des années 1960 m’apparaissaient comme les lieux de regards éparpillés, s'incarnant dans un corps collectif issu de la campagne ou de la 
périphérie de Tokyo, et pullulant dans ces quartiers, le «Ginza» postérieur aux années 1920, comme le "Shibuya» postérieur aux années 1970 étaient quant à eux autant de scènes où les jeunes populations jouaient leur propre rôle sous le regard systémique de l'État, du capital et des médias. De là, je mettais en relief la dualité de la «modernité» tokyoïte, en d'autres termes, la dualité d'une "modernité» telle que vécue, d'une part, par une pluralité de corps assemblés dans les villes, et telle qu'organisée, d'autre part, à travers le regard moderne.

À partir des années 1990, l'analyse de Tokyo comme «lieu de regards» s'est poursuivie grâce au travail de nombreux sociologues. Par exemple, dans Kökoku toshi - Tökyō『広告都市・東京』(La ville-publicité - Tokyo), Kitada Akihiro 北田暁大 a montré comment les procédés qui permettaient de recouvrir sémiotiquement l'espace métropolitain, comme à «Shibuya» jusqu'aux années 1980, sont subitement devenus caducs à partir des années 1990, avec la transformation des médias et du capital. Pour Kitada,

la logique de la ville-publicité, qui invitait le passant dans un espace de consommation en recouvrant la ville de publicités "fantômatiques ", c'est-à-dire en la théâtralisant, pour mieux promouvoir l'identité Shibuya dans sa dimension culturelle de pointe, est devenue caduque à cause des regards [dé-théâtralisés] "post-années 1980" des passants qui, sans plus chercher aucune théâtralité dans la ville, appréhendaient Shibuya [simplement] comme un "vaste quartier parmi d'autres». (Kitada 2012 : 127)

À partir des années 1990, les passants à Tokyo sont caractérisés par «un comportement qui consiste, non pas à "lire" la ville comme une œuvre littéraire ou une série télévisée (comme un texte), mais plutôt à la "voir défiler" (minagasu 見流す) comme un film publicitaire” (Kitada 2012 : 128). Une ville gagnée par ce type d'attitudes n'autorise plus aucun «travail de montage à même d'ordonner [les différents éléments d'un lieu ou d'un espace] en un texte ou une représentation dotée d'un sens symbolique déterminé». Au contraire, depuis les années 1990,

l'espace réel dénommé Shibuya [est] une bibliothèque d'informations à forte teneur en "choses intéressantes» ou "à posséder» dans l'optique de liens avec autrui, pareille, autrement dit, à un gigantesque convenience store (kombini コンビニ), et dont le paysage [s'est changé en] une matière première à ponctionner librement grâce à la caméra numérique intégrée à son portable. (Kitada 2012 : 165-166) 
Abstraction faite de sa comparaison avec une «bibliothèque», l'observation de Kitada fait mouche : à partir des années 1990, la ville a cessé d'être "lisible» pour relever davantage du "zapping».

La phase correspondant à l'existence d'une "ville-publicité» connaît des mutations : en d'autres termes, la logique publicitaire s'oppose désormais à la formation concrète de la ville, de sorte qu'il devient difficile de mettre publicitairement en scène ou en récit chaque "quartier». Mais alors les "regards", qui dès le départ s'étaient définis dans leur dimension collective, peuvent-ils continuer d'exister comme tels? D’un autre côté, s'il émet l'hypothèse que la ville puisse être devenue un «fantôme culturel» au sens où l'entend [Marshall] MacLuhan, Kitada refuse de reconnaître un destin semblable pour la publicité. Il met plutôt en évidence

l'«instinct de décontextualisation ou de parasitisme médiatique grâce auquel la publicité, dépourvue de corps institué, s'agrège à des médias multiples pour troubler sans cesse l'ordre contextuel du monde normal ", et l'oblige à définir les contours de son nouveau corps dès qu'elle prend acte de la caducité de sa méthodologie urbaine. (Kitada 2012 : 171)

Dans ces conditions, la «ville», délaissée par cette "publicité» désormais dissociée, saurait-elle conserver son épaisseur et sa dimension collective en tant que lieu, si elle se définit comme "fantôme culturel»? En d'autres termes, si la Tokyo des années 1980 a incarné de manière flagrante le schéma "publicité $\rightarrow$ ville», cette "ville» dissociée de la "publicité» est-elle nécessairement devenue un cimetière de carcasses et de fantômes?

Ici, les considérations de Wakabayashi Mikio 若林幹夫 sur ville et regard nous permettent de déterminer pour l'espace urbain de Tokyo la position historique des mutations examinées par Kitada, propres aux années 1990. Se référant à la théorie des espaces homogènes proposée par l'architecte Hara Hiroshi 原広司, Wakabayashi avance que la «modernité», fondamentalement, contenait en elle l'éventualité d'une "émancipation vis-à-vis de l'espace». Un «espace homogène» est "un espace homogénéisé tant physiquement que socialement, capable de s'adapter avec souplesse à tous les usages, tant il s'est libéré des éléments matériels et culturels qui contraignaient justement sa structure et son usage». Soit dit en passant, faire de ce type d'espace l'horizon fondamental de la ville et de ses bâtiments est l'ultime "solution» à laquelle ont abouti les recherches de l'architecture 
moderne. Concept permettant à la société moderne de s'émanciper ellemême des normes spatiales qui conditionnaient les sociétés du passé, un espace homogène signifie qu'ainsi «l'homme, sujet capable d'agir par lui-même, libre de toute transcendance comme de toute métaphysique, devient la nouvelle instance à même de donner un sens à l'espace qu'il s'est subordonné» (Wakabayashi 2003 : 38-43).

Ainsi en va-t-il exactement : l'espace homogène voit apparaître (en même temps qu'il est soutenu par) des réseaux de communication et de circulation à grande vitesse, sert d'intermédiaire aux désirs et aux rêves des gens autant qu'aux mouvements du capitalisme, et se place ainsi aux fondements d'une ville contemporaine structurée en tours et gratte-ciel. C'est-à-dire un processus au cours duquel «la ville qui [dans les sociétés prémodernes] avait fonctionné comme un médium régissant les actes et les relations des hommes entre eux, forte d'un symbolisme et d'une systématicité totalisantes, a bientôt cessé de fonctionner de la sorte» (Wakabayashi 2003 : 48).

Ainsi la ville en tant qu'ordre spatial symbolique a-t-elle continué de se pulvériser tout au long de l'époque moderne. Ce qu'il en reste aujourd'hui, ce sont sa dépouille, ses ruines, son spectre errant. Dans ces conditions, la "ville/publicité» des années 1980 n'était peut-être alors en soi qu'un fantôme fabriqué par les médias et les stratégies marketing de l'époque.

En fondant son concept sur cet espace homogène, résume Wakabayashi, le contemporain a vu l'espace urbain perdre progressivement les caractères de totalisation et de système propres à la ville traditionnelle, pour ne plus produire que les images fictives de lui-même, à mesure que s'effondraient les unes dans les autres ses opérations sémiotiques et le media-mix qu'il formait avec les autres médias, et notamment les médias de masse. (Wakabayashi 2003 : 53)

Dans la Tokyo des années 1990, cette stratégie de sémiotisation de la ville qu’il qualifie de "télévisuelle» est tombée en désuétude. Mais même une fois révélées les limites de cette ville «télévisuelle» ou encore "parcà-thématique» (têmapākuteki テーマパーク的), le processus, essentiellement de long terme, qui voit l'ordre symbolique de la ville se démanteler radicalement, ne s'achève pas pour autant. Indissolublement lié au capitalisme, il continue de traverser de part en part la ville contemporaine, même si le temps est révolu d'une mise en scène sémiotique/textuelle de l'urbain. 


\section{Ville-Disneyland et géopolitique du regard}

À côté de cette thèse, une autre avance que les villes "publicité [ou parcà-thématiques] », apparues dans les années 1980, ont continué de proliférer au Japon même après les années 1990. Dans Dizunirandoka suru shakai de kibō wa ikani katariuru ka? 『ディズニーランド化する社会で希望はいかに語り うるか』(Comment dire le désir dans une société en cours de disneylandisation?), Hasegawa Hajime 長谷川一 adopte cet autre point de vue : après le dégonflement de la bulle, la «disneylandisation» aurait d'autant plus largement gagné la société japonaise. Ainsi,

dans cette société changée en média postmoderne de consommation de l'information (jōhô shōhi shakai 情報消費社会), image et consommation sont profondément liées dans toutes les activités du quotidien. Y prenant ses racines, l'anormalité à-laDisneyland pénètre largement toutes les couches du monde normal et [ne cesse] d'y croître.

Aujourd'hui, «le même procédé qui, à Disneyland, vous procure une impression d'anormalité joyeuse mêlée d'exaltation, se retrouve exactement dans le quotidien, qu'il recouvre hermétiquement comme à l'aide d'un épais calfeutrage» (Hasegawa 2014 : 3-5). Dans la mesure où Disneyland et l'excès de sens qui le caractérise auraient pénétré toutes les couches de la société, le «regard déthéâtralisé» décrit par Kitada se comprend alors comme la conséquence d'une «société qui cesse d'être perçue comme porteuse de sens, et, en tant que telle, n'a plus besoin d'extériorité». De même, le renversement d'une ville «disneylandisée et submergée de sens» en une ville [bâtie sur] «une "socialité de la connexion” (tsunagari no shakaisei つな がりの社会性), où s'est perdu tout intérêt pour le sens, détrôné par la communication pour la communication", se comprend comme la conséquence d'un «système sémiotique autonomisé jusqu'à l'autosuffisance» (Hasegawa 2014 : 42-43).

De toute évidence, pareille absentéisation du «dehors» décrit le moment (momento モメント) auquel la société actuelle est désormais gagnée dans son entier. Et si une «forme extrême de complétude, hermétique et coupée du dehors", caractérise en premier lieu Disneyland, alors résumer les tendances de fond de la ville contemporaine du terme de "disneylandisation" n'est pas impossible. De fait, les hypothèses que j'ai moi-même avancées naguère 
se superposaient à la thèse de Hasegawa [...]. Dans cette perspective, alors qu’ils avaient absorbé (shōhi shiteita 消費していた) jusqu’aux années 1980 le récit d'une "ville sans dehors" que les Disney et autres Parco leur soumettaient à Urayasu ou Shibuya, à partir des années 1990, les individus en sont eux-mêmes devenus les énonciateurs innombrables. Caractérisant pareille continuité par la prolifération des «attractions», qui mettent en avant "participation» (sanka 参加) et "expérience» (taiken 体験), Hasegawa découvre ainsi l'ambivalence des possibilités ouvertes par leur extension technique. Les «attractions» ressortissent à ces activités auxquelles le corps peut directement prendre part. Dans sa dimension immédiate et interactive, la disneylandisation contient donc en soi la possibilité d'aller contre son propre principe. D'un autre côté, quand Kitada introduit dans la ville publicitaire cette coupure du "regard déthéâtralisé», ce qu’il vise est moins l'implication des corps que l'activité du «capital». Car sans y être radicalement extérieur, le «capital» n'appartient pas non plus pour autant à la ville-publicité telle que nous la vivons. Il ne révèle pas le «dehors» de notre monde, mais élargit la zone où nos activités entrent en contact avec lui.

Revenons donc à la thèse de Kitada : dans Truman Show, le film qu'il prend pour exemple $e^{5}$ la "publicité» apparaît comme «le motif qui fait avancer l'histoire de Truman, ce motif primordial qui suggère à Truman qu'il puisse exister un "dehors" ". La ville y implique une organisation où "intérieur [fiction] et extérieur [capital] se lient l'un à l'autre grâce à la publicité». En d'autres termes, celle-ci est pour la ville comme un ruban de Mœbius : en tant que medium continuant, selon les circonstances, de mettre en scène ou de construire le monde intérieur, c'est-à-dire une fiction, elle a précisément pour fonction de renvoyer à un « réel [= au capital], qui en soi ne saurait être sémiotiquement fermé» (Kitada 2012 : 12).

Incontestablement, tandis qu'avec la modernité l'ensemble de l'espace social s'est homogénéisé, l'intériorité de la ville, constituée de façon secondaire et préservée comme telle, s'est vue continûment disloquée et fictionnalisée. Les expositions et les grands magasins, les salles de cinéma, mais aussi les espaces autour des téléviseurs publics ou les parcs à thèmes, qui à partir du XIX ${ }^{e}$ siècle se sont insérés dans la ville, ont été conçus à l'origine

5. Cf. The Truman show, film de Peter Weir sorti en 1998. Incarné par Jim Carrey, Truman Burbank y est à son insu le protagoniste d'un gigantesque reality show. (NdT) 
comme les lieux d'une foule moderne, c'est-à-dire fictive. Et dans le plan de ces espaces, l'organisation des «regards» a joué un rôle décisif. Le capitalisme moderne n'a cessé d'organiser ainsi non seulement les lieux directement disciplinaires ou d'instruction tels que prisons, usines ou écoles, mais aussi, dans ces lieux visuels de rassemblement, les relations entre le «capital» et les «corps».

Dans ces conditions, il est désormais nécessaire de décrire la dynamique de stratification de la "vision", telle que vécue en divers lieux des "villes» modernes et contemporaines, comme l'histoire d'une expérience sociale née à la frontière entre réalité et fiction, entre "dedans" et "dehors». J'ai affirmé jusque-là que la ville moderne était à la fois "prison " et "théâtre». Aux sens de [Michel] Foucault et de [Jonathan] Crary, prison et théâtre ont en commun d'être des lieux «visuels». Toutefois, dans cette ville envisagée comme théâtre, c'est-à-dire dans la ville perçue comme un tableau directement connecté au capitalisme, «regarder» et «être regardé» ne s'opposent pas comme à l'intérieur d'un panoptique : au contraire, ces expériences sont comme les deux faces des pratiques individuelles et collectives. C'està-dire des techniques de «représentation théâtrale» (jöen 上演) plus que de "surveillance». Ces « représentations» sont multiples, afin de répondre à la distribution géographique des événements comme aux situations environnementales (bâtiments et moyens de transports, truchement des médias), mais aussi profondément liées aux caractéristiques sociales de ceux qui les portent (classe et genre, âge, ethnicité). Si l'on prend comme unité la capitale impériale ou la ville globale à telle ou telle période, apparaissent des similarités entre les expériences qu'on peut y faire respectivement, de sorte qu'il semble possible de les décrire en bloc. Toutefois, cette communauté résulte d'un simple processus d'intégration déterminé, c'est-à-dire de la superposition des interactions visuelles jouées ou incarnées dans des endroits très divers. C'est pourquoi notre réflexion doit plutôt, au-delà de ces ressemblances structurales intégrées, conserver un point de vue qui revienne au niveau de chaque lieu en sa réalité concrète.

Traduit du japonais et annoté par Mathieu CAPEL. 


\section{Bibliographie}

FRIEDBERG ANNE アン•フリードバーグ 2008

Uindō shoppingu - eiga to posutomodan ウインドウショッピングー映画とポスト モダン (Lèche-vitrine - cinéma et postmoderne), Shōhakusha 松柏社, Tokyo. La traduction se fonde ici sur l'ouvrage original en anglais, Window Shopping Cinema and the Postmodern, Berkeley, University of California Press, 1993.

\section{FRIEDBERG Anne アン•フリードバーグ} 2012

Vācharu uindō-Aruberuti kara

Maikurosofuto made ヴァーチャル・ウィンド ウ一アルベルティからマイクロソフトまで (La fenêtre virtuelle - d'Alberti à Microsoft), Tokyo, Sangyōtosho 産業図書.

La traduction se fonde ici sur l'ouvrage original en anglais, The Virtual Window - From Alberti to Microsoft, Cambridge, MIT Press, 2009.

\section{HASEGAWA Hajime 長谷川- 2014} Dizunīrandoka suru shakai de kibō wa ikani katariuru ka - tekunorojī to shintai no yūgi ディズニーランド化する社会で希望 はいかに語りうるか - テクノロジーと身体 の遊戯 (Comment dire le désir dans une société en cours de disneylandisation? Le jeu de la technologie et du corps), Tokyo, Keiō gijuku daigaku shuppankai 慶應義塾大学出版会.

KITADA Akihiro 北田暁大 2012

Kōkoku toshi-Tōkyō 広告都市・東京: その誕生と死 (La ville-publicité - Tokyo : sa naissance, sa mort), Tokyo, Kōsaidō shuppan 廣済堂出版.

NAKAMURA Hideyuki 中村秀之 2010 Gareki no tenshitachi-Benyamin kara " eiga » no mihatenu yume e 瓦礫の天使 たち一ベンヤミンから“映画”の見果てぬ 夢へ (Les anges des gravats - de Benjamin aux rêves sans fin du «cinéma»), Tokyo, Serika shobō せりか書房.

\section{ŌKUBO Ryō 大久保遼 2015}

Eizō no arukeorojī : shikaku riron, kōgaku media, eizō bunka 映像のアルケオロジー : 視覚理論・光学メディア・映像文化 (Archéologie des images - logique visuelle, médias optiques, culture des images), Tokyo, Seikyūsha 青弓社, coll. Shikaku bunka sōsho 視覚文化叢書, n4.

WAKABAYASHI Mikio 若林幹夫 2003 Toshi e/kara no shisen 都市へ/からの 視線 (Regarder la ville/de la ville), Tokyo, Seikyūsha 青弓社.

YOSHIMI Shun.ya 吉見俊哉 1987 Toshi no doramaturugi : Tōkyō, sakariba no shakaishi 都市のドラマトゥルギー一東京・ 盛り場の社会史 (La dramaturgie de la ville - Tokyo, histoire sociale de ses quartiers animés), Tokyo, Kōbundō 弘 文堂

\section{YOSHIMI Shun.ya 吉見俊哉 1992}

Tenrankai no seijigaku: manazashi no kindai 展覧会の政治学一眼差しの近代 (Politique de l'exposition - modernité du regard), Tokyo, Chūkō shinsho 中公 新書. 
